\title{
RARRES3-suppressing metastases to the lung in breast cancer
}

About $90 \%$ of deaths due to breast cancer are caused by distant metastases. Oestrogen receptor (ER)-negative breast cancer tumours account for $20-30 \%$ of breast cancer cases, which primarily metastasize to the lungs. A group led by Roger Gomis has recently demonstrated that loss of function of RARRES3, a retinoic acid responsive gene, in ER-negative breast cancer cells stimulates their invasive capacity and promotes metastasis to the lung.

Gomis explains, "the transformation of a tumour cell into a metastatic cell is not just about acquiring capacities, but equally important is the loss of certain genes, such as RARRES3." The researchers combined studies in mouse models and cell lines, and ultimately validated their findings in 580 samples from primary breast tumours, demonstrating that RARRES3 is suppressed in ER-negative breast tumours. Furthermore, RARRES3 loss is associated with a lack of differentiation capacity, which facilitates the initiation of metastases. Therefore, RARRES3 loss could provide a marker to identify patients with greater susceptibility to lung metastasis.

"We are currently exploring the potential clinical implications of our findings," says Gomis. However, the researchers believe that some patients may benefit from treatment with retinoic acid after the removal of the original tumour, to induce RARRES3 expression, enhance cell differentiation and prevent metastases.

Alessia Errico

Original article Morales, M. et al. RARRES3 suppresses breast cancer lung metastasis by regulating adhesion and differentiation. EMBO Mol. Med. doi:10.15252/ emmm.201303675 